\title{
THE TREE PROPERTY AND THE FAILURE OF SCH AT UNCOUNTABLE COFINALITY
}

\author{
DIMA SINAPOVA
}

\begin{abstract}
Given a regular cardinal $\lambda$ and $\lambda$ many supercompact cardinals, we describe a type of forcing such that in the generic extension there is a cardinal $\kappa$ with cofinality $\lambda$, the Singular Cardinal Hypothesis at $\kappa$ fails, and the tree property holds at $\kappa^{+}$.
\end{abstract}

\section{INTRODUCTION}

The relationship between the singular cardinal hypothesis ( $\mathrm{SCH}$ ), square principles, the tree property and large cardinals is important in singular cardinal arithmetic. The tree property at $\kappa^{+}$states that there are no Aronszajn trees at $\kappa^{+}$i.e. that every $\kappa^{+}$-tree has an unbounded branch. Recently an old question was answered by Neeman [5] in the negative: whether failure of SCH implies the existence of an Aronszajn tree. Previously the only known way to establish the tree property at a successor of a singular cardinal was due to Magidor-Shelah [4].

The result in Neeman [5] that the failure of SCH is consistent with the tree property was obtained at a cardinal of cofinality $\omega$. Here we show that the failure of $\mathrm{SCH}$ is consistent with the tree property for cardinals of arbitrary cofinality.

Theorem 1. Suppose that $\lambda$ is a regular uncountable cardinal, $\left\langle\kappa_{\alpha} \mid \alpha<\lambda\right\rangle$ is a continuous sequence such that $\lambda<\kappa_{0}, \kappa_{0}$ and each $\kappa_{\alpha+1}$ are supercompact cardinals and let $\nu=\sup _{\alpha} \kappa_{\alpha}$. Then there is a generic extension in which:

(1) $\kappa=\kappa_{0}$ is preserved and has cofinality $\lambda$,

(2) the tree property holds at $\kappa^{+}$and SCH fails at $\kappa$,

(3) there is a very good scale and a bad scale at $\kappa$.

The rest of the paper presents the proof of Theorem 1. In section 2 we define the forcing notion and give some basic properties about the forcing. The forcing that we will use combines ideas from Neeman [5] and Sinapova [6]. Also, we describe the very good scale and the bad scale in the generic extension. Scales are a central concept in PCF theory. The relationship between scales, the $\mathrm{SCH}$, and square principles and singular arithmentic has been explored by Gitik, Cummings, Foreman, Magidor among others. In 2008 Gitik-Sharon [2] showed two important consistency results about scales: that failure of SCH does not imply weak square, and the existence of 
a very good scale does not imply weak square. The result was generalized by Sinapova [6] for singular cardinals of arbitrary cofinality. The Gitik-Sharon model provided much of the motivation behind the construction in Neeman [5]. Finally in section 3 we prove that the tree property holds. Both in the $\omega$ cofinality case and the uncountable cofinality case it remains open whether the result can be pushed down to small cardinals.

\section{The CONSTRUCtion}

Let $\left\langle\kappa_{\xi} \mid \xi<\lambda\right\rangle$ be a continuous increasing sequence, such that each $\kappa_{\xi+1}$ is supercompact and $\lambda<\kappa_{0}$. Denote $\kappa=\kappa_{0}$. Let $\nu=\sup _{\xi<\lambda} \kappa_{\xi}$ and $\mu=\nu^{+}$. Using Laver's preparation, we may assume that the supercompactness of $\kappa$ is indestructible under $\kappa$-directed closed forcing [3]. Let $E$ be $A d d\left(\kappa, \nu^{++}\right)$ generic over $V$. Work in $V[E]$.

Proposition 2. There is a sequence $\left\langle U_{\xi} \mid \xi<\lambda\right\rangle$, where each $U_{\xi}$ is a normal measure on $\mathcal{P}_{\kappa}\left(\kappa_{\xi}\right), \kappa$ is $\kappa_{\xi}$-supercompact in $U l t\left(V[E], U_{\xi}\right)$, and the sequence is Mitchell order increasing, i.e. for $\xi<\eta<\lambda, U_{\xi} \in U l t\left(V[E], U_{\eta}\right)$, and there are functions $\left\langle f_{\gamma}^{\xi} \mid \gamma<\mu, \xi<\lambda\right\rangle$ from $\kappa$ to $\kappa$, such that for all $\xi<\lambda, \gamma<\mu, j_{U_{\xi}}\left(f_{\gamma}^{\xi}\right)(\kappa)=\gamma$.

Proof. We use the following claim:

Claim 3. For all $\xi<\lambda$, for all $\mathcal{X} \subset \mathcal{P}\left(\mathcal{P}_{\kappa}\left(\kappa_{\xi}\right)\right)$, there is a normal measure $U_{\xi}$ on $\mathcal{P}_{\kappa}\left(\kappa_{\xi}\right)$, such that $\mathcal{X} \in U l t\left(V[E], U_{\xi}\right)$, and there are functions $\left\langle f_{\gamma}\right|$ $\gamma<\mu\rangle$ from $\kappa$ to $\kappa$, such that for all $\gamma<\mu, j_{U_{\xi}}\left(f_{\gamma}\right)(\kappa)=\gamma$.

Proof. The proof of this claim adapts an argument due to Solovay, Reinhardt, and Kanamori [7]. For details, see Lemma 2 in [6].

Now, define the chain as follows. Suppose that we already have $\left\langle U_{\eta}\right|$ $\eta<\xi\rangle$ and $\left\langle f_{\gamma}^{\eta} \mid \gamma<\mu, \eta<\xi\right\rangle$ as desired. Let $\bar{U}$ be a normal measure on $\mathcal{P}_{\kappa}\left(\kappa_{\xi}\right)$. We can code $\bar{U}$ and the $U_{\eta}$ 's by some $\mathcal{Y} \subset \mathcal{P}\left(\mathcal{P}_{\kappa}\left(\kappa_{\xi}\right)\right)$. Apply the claim to find a normal measure $U_{\xi}$ on $\mathcal{P}_{\kappa}\left(\kappa_{\xi}\right)$ with $\mathcal{Y} \in U l t\left(V[E], U_{\xi}\right)$ and functions $\left\langle f_{\gamma}^{\xi}\right| \gamma\langle\mu\rangle$ from $\kappa$ to $\kappa$ with $j_{U_{\xi}}\left(f_{\gamma}^{\xi}\right)(\kappa)=\gamma$ for each $\gamma$. Then $\bar{U} \in U l t\left(V[E], U_{\xi}\right)$, and so $\kappa$ is $\kappa_{\xi}$-supercompact in $U l t\left(V[E], U_{\xi}\right)$.

Fix measures $U_{\xi}$, for $\xi<\lambda$ and functions $\left\langle f_{\gamma}^{\xi} \mid \gamma<\mu, \xi<\lambda\right\rangle$ as in the statement of the last proposition. For $\xi<\lambda$, let $X_{\xi}$ be the set of $x \in \mathcal{P}_{\kappa}\left(\kappa_{\xi}\right)$ such that

(1) $x \cap \kappa={ }_{\text {def }} \kappa_{x}$ is an ordinal, $\lambda<\kappa_{x}$, and $\kappa_{x}$ is $f_{\kappa_{\xi}}^{\xi}\left(\kappa_{x}\right)$-supercompact,

(2) $(\forall \eta \leq \xi)$ o.t. $\left(x \cap \kappa_{\eta}\right)=f_{\kappa_{\eta}}^{\xi}\left(\kappa_{x}\right)$,

(3) $(\forall \eta \leq \xi)\left(f_{\kappa_{\eta}}^{\xi}\left(\kappa_{x}\right)^{<\kappa_{x}} \leq f_{\kappa_{\eta}^{+}}^{\xi}\left(\kappa_{x}\right)\right)$.

By standard reflection arguments $X_{\xi} \in U_{\xi}$. Here clause (3) is due to reflection of $\kappa_{\eta}^{<\kappa}=\kappa_{\eta}$ when $\eta$ is a successor, and $\kappa_{\eta}^{<\kappa}=\kappa_{\eta}^{+}$for $\eta$ limit. Note that for $\eta<\eta^{\prime}<\lambda$, if $x \in X_{\eta^{\prime}}$, then $x \cap \kappa_{\eta} \in X_{\eta}$. 
For $\xi<\eta<\lambda$, for $x \in X_{\eta}$, and $Y \subset \mathcal{P}_{\kappa_{x}}\left(\kappa_{\xi} \cap x\right)$, define $\bar{Y} \subset \mathcal{P}_{\kappa_{x}}$ (o.t. $(x \cap$ $\left.\left.\kappa_{\xi}\right)\right)$, by $\bar{Y}=\{\{$ o.t. $(\delta \cap x) \mid \delta \in y\} \mid y \in Y\}$. Since $U_{\xi} \in U l t\left(V[E], U_{\eta}\right)$, there is a function $x \mapsto \overline{U_{\eta, x}^{\xi}}$, such that $U_{\xi}=\left[x \mapsto \overline{U_{\eta, x}^{\xi}}\right]_{U_{\eta}}$ and each $\overline{U_{\eta, x}^{\xi}}$ is a normal measure on $\mathcal{P}_{\kappa_{x}}\left(f_{\kappa_{\xi}}^{\eta}\left(\kappa_{x}\right)\right)=\mathcal{P}_{\kappa_{x}}\left(\right.$ o.t. $\left.\left(x \cap \kappa_{\xi}\right)\right)$. We lift this measure to a normal measure $U_{\eta, x}^{\xi}$ on $\mathcal{P}_{\kappa_{x}}\left(\kappa_{\xi} \cap x\right)$. In particular, $\overline{U_{\eta, x}^{\xi}}=\{\bar{Y} \subset$ $\left.\mathcal{P}_{\kappa_{x}}\left(f_{\kappa_{\xi}}^{\eta}\left(\kappa_{x}\right)\right) \mid Y \in U_{\eta, x}^{\xi}\right\}$. Note that each $U_{\eta, x}^{\xi}$ is $\lambda$ - complete. For $\theta<\lambda$, let $B_{\theta}=\left\{z \in X_{\theta} \mid(\forall \xi, \eta) \xi<\eta<\theta \rightarrow \overline{U_{\theta, z}^{\xi}}=\left[x \mapsto \overline{U_{\eta, x}^{\xi}}\right]_{U_{\theta, z}^{\eta}}\right\}$. Adapting the arguments in [6] we have that each $B_{\theta} \in U_{\theta}$.

We are ready to define the main forcing $\mathbb{P}$. Conditions are of the form $p=\langle g, H\rangle$, where:

(1) $\operatorname{dom}(g)$ is a finite subset of $\lambda$ and $\operatorname{dom}(H)=\lambda \backslash \operatorname{dom}(g)$,

(2) for each $\xi \in \operatorname{dom}(g), g(\xi) \in B_{\xi}$,

(3) for $\xi<\eta$, in $\operatorname{dom}(g)$, we have $g(\xi) \prec g(\eta)$ i.e. $g(\xi) \subset g(\eta)$ and o.t. $(g(\xi))<\kappa_{g(\eta)}=\kappa \cap g(\eta)$

(4) for $\xi \notin \operatorname{dom}(g)$ and $\xi>\max (\operatorname{dom}(g))$, we have $H(\xi) \in U_{\xi}, H(\xi) \subset$ $B_{\xi}$

(5) for $\xi \notin \operatorname{dom}(g)$ and $\xi<\max (\operatorname{dom}(g))$, setting $\eta=\min (\operatorname{dom}(g) \backslash \xi)$, we have $H(\xi) \in U_{\eta, g(\eta)}^{\xi}$ (the normal measure on $\left.\mathcal{P}_{\kappa_{g(\eta)}}\left(\kappa_{\xi} \cap g(\eta)\right)\right)$,

(6) for $\xi<\eta$, if $\xi \in \operatorname{dom}(g), \eta \notin \operatorname{dom}(g)$, then for each $z \in H(\eta)$, $g(\xi) \prec z$.

We say that $g$ is the stem of $p$.

$\langle g, H\rangle \leq\langle j, J\rangle$ iff

(1) $g \supset j$,

(2) for $\xi \in \operatorname{dom}(g) \backslash \operatorname{dom}(j), g(\xi) \in J(\xi)$,

(3) for $\xi \notin \operatorname{dom}(g)$, we have $H(\xi) \subset J(\xi)$.

If $q \leq p$ and both conditions have the same stem, we say that $q$ is a direct extension of $p$ and write $q \leq^{*} p$.

$\mathbb{P}$ is a combination of the forcing notions from [5] and [6]. Adapting the arguments in [6] we get:

Proposition 4. (Properties of the forcing notion)

(1) $\mathbb{P}$ has the $\nu^{+}$chain condition (since any two conditions with the same stem are compatible and the number of possible stems is $\nu$ ).

(2) $\mathbb{P}$ satisfies the Prikry property, i.e. if $\phi$ is a formula and $p$ is a condition, then there is $q \leq^{*} p$, such that $q$ decides $\phi$.

(3) A corollary to the above is that if $\phi$ is a formula, $p=\langle g, H\rangle$ is a condition and $\alpha \in \operatorname{dom}(g)$, then there is $q \leq^{*} p$, such that if $r \leq p$ decides $\phi$, then $r \nmid \alpha \neg q \uparrow(\lambda \backslash \alpha)$ decides $\phi$. Here we use that $\mathbb{P}_{\alpha}^{p}=\{r|\alpha| r \leq p\}$ has size less than $\kappa_{g(\beta)}$, where $\beta=$ $\min (\operatorname{dom}(g) \backslash \alpha+1)$. All the measures used in $p$ above $\alpha$ are $\kappa_{g}(\beta)$ complete, so we can apply the Prikry property to every element in $\mathbb{P}_{\alpha}^{p}$ and then intersect measure one sets. 
Let $G$ be $\mathbb{P}$ generic and let $g^{*}=\bigcup_{\langle g, H\rangle \in G} g$. Then $g^{*}$ is a function with domain $\lambda$ and with $g^{*}(\alpha) \in \mathcal{P}_{\kappa}\left(\kappa_{\alpha}\right)$ for each $\alpha \in \lambda$. For each $\alpha<\lambda$, denote $x_{\alpha}^{*}=g^{*}(\alpha)$ and $\tau_{\alpha}=\kappa_{x_{\alpha}^{*}}=\kappa \cap x_{\alpha}^{*}$. Then $\nu=\bigcup_{\alpha<\lambda} x_{\alpha}^{*}$, and so the cofinality of $\kappa$ and each $\kappa_{\alpha+1}$ is $\lambda$. In particular, in the generic extension $\kappa=\sup _{\alpha<\lambda} \tau_{\alpha}$. Below we summarize the preservation and collapsing of cardinals and cofinalities. The proof is an adaptation of the arguments in $[6]$.

Proposition 5. (Preservation of cardinals)

(1) $\kappa$ is preserved and has cofinality $\lambda$ in the generic extension.

(2) All cardinals and cofinalities below $\tau_{0}$ are preserved.

(3) For each $\alpha<\lambda, \tau_{\alpha}$ is preserved.

(4) Let $\tau$ be a cardinal in $V$ with $\tau_{\alpha}<\tau \leq \sup _{\xi<\alpha} f_{\kappa_{\xi}}^{\alpha}\left(\tau_{\alpha}\right)$, for $\alpha$ limit. Then $\operatorname{card}^{V[E][G]}(\tau)=\tau_{\alpha}$, and if $\tau$ is regular in $V$, then in $V[E][G]$ the cofinality of $\tau$ is equal to $\operatorname{cf}(\alpha)$.

(5) Let $\tau$ be a cardinal in $V$ with $\kappa<\tau<\mu$. Then $\operatorname{card}^{V[E][G]}(\tau)=\kappa$, and if $\tau$ is regular in $V$, then in $V[E][G]$ the cofinality of $\tau$ is equal to $\lambda$.

(6) Cardinals greater than or equal to $\mu$ are preserved. And so $\mu$ becomes the successor of $\kappa$.

Remark 6. In particular, if $\tau$ is such that $\operatorname{cf}^{V}(\tau) \neq \operatorname{cf}^{V[E][G]}(\tau)$, then $\operatorname{cf}^{V[E][G]}(\tau) \leq$ $\lambda$.

The reason behind item (4) is as follows: let $p=\langle g, H\rangle \in G$ with $\operatorname{dom}(g)=\{\alpha\}$ for a limit $\alpha<\lambda$. Below this condition we can factor the poset to $\mathbb{P}_{0} \times \mathbb{P}_{1}$, where $\mathbb{P}_{0}$ is defined from the normal measures $\overline{U_{\alpha, x_{\alpha}^{*}}^{\xi}}$ on $\mathcal{P}_{\tau_{\alpha}}\left(f_{\kappa_{\xi}}^{\alpha}\left(\tau_{\alpha}\right)\right)$ for $\xi<\alpha$ and $\mathbb{P}_{1}$ is defined from the normal measures $U_{\beta}$, $\alpha<\beta<\lambda$. I.e. conditions in $\mathbb{P}_{1}$ are below $\langle 0, H \uparrow(\lambda \backslash \alpha)\rangle$. Then $\mathbb{P}_{0}$ adds a generic sequence $\left\langle y_{\xi}\right| \xi\langle\alpha\rangle$, such that $\bigcup_{\xi<\alpha} y_{\xi}=\sup _{\xi<\alpha} f_{\kappa_{\xi}}^{\alpha}\left(\tau_{\alpha}\right)$. Thus $\sup _{\xi<\alpha} f_{\kappa_{\xi}}^{\alpha}\left(\tau_{\alpha}\right)$ is collapsed to $\tau_{\alpha}$. Moreover, $\mathbb{P}_{0}$ has the $\sup _{\xi<\alpha} f_{\kappa_{\xi}}^{\alpha}\left(\tau_{\alpha}\right)^{+}$ chain condition. For more details on this factoring see [6].

The factoring described above combined with the Prikry property gives that for limit $\alpha<\lambda$ and natural number $k$, cardinals $\tau$ with $\sup _{\xi<\alpha} f_{\kappa_{\xi}}^{\alpha}\left(\tau_{\alpha}\right)^{+} \leq$ $\tau<\tau_{\alpha+k}$ are preserved. This implies item (3) of the above proposition.

Corollary 7. In the generic extension, $2^{\kappa}=\kappa^{++}$, and so the singular cardinal hypothesis fails at $\kappa$.

Before we focus on the tree property, we turn our attention to scales in the generic extension. Scales are a central concept in PCF theory. The existence of a bad scale implies the failure of weak square. Actually it also implies that the approachability property fails. In both the models of Gitik-Sharon [2] and Neeman [5] there exists a very good scale and a bad scale. Gitik-Sharon [2] showed that starting from a supercompact there is a generic extension, in which the singular cardinal hypothesis fails at $\aleph_{\omega^{2}}$, 
there is a very good scale at $\aleph_{\omega^{2}}$ and the approachability property fails at $\aleph_{\omega^{2}}$. The proof of the existence of the bad scale in the Gitik-Sharon model is due to Cummings-Foreman [1]. The construction in [2] was generalized to a cardinal of arbitrary cofinality by Sinapova [6]. Adapting the arguments from the above papers, we get:

Proposition 8. In $V[E][G]$, there is a bad scale and a very good scale at $\kappa$.

As before, denote $\mu=\left(\nu^{+}\right)^{V}$. Using that $\kappa$ is supercompact, in $V[E]$ we fix a bad scale $\left\langle G_{\beta} \mid \beta<\mu\right\rangle$ in $\prod_{\xi<\lambda} \kappa_{\xi}^{+}$. Moreover we can fix $\lambda<\tau<\kappa$, such that there is a stationary set of bad points of cofinality $\tau$. We can define the forcing so that the generic sequence $\left\langle\tau_{\xi} \mid \xi<\lambda\right\rangle$ is above $\tau$, and all of the measures are $\tau$-complete. When defining the scales, we use the following key property (due to a density argument):

Proposition 9. If $\left\langle A_{\xi} \mid \xi<\lambda\right\rangle \in V[E]$ is such that each $A_{\xi} \in U_{\xi}$, then $x_{\xi}^{*} \in A_{\xi}$ for all sufficiently large $\xi$.

We also make use of a bounding lemma. For details of the proof, see [6].

Lemma 10. (Bounding)

(1) Suppose that in $V[E][G], f \in \prod_{\xi<\lambda} f_{\kappa_{\xi}^{+}}^{\xi}\left(\tau_{\xi}\right)$. Then there is a sequence of functions $\left\langle H_{\eta} \mid \eta<\lambda\right\rangle$ in $V[E]$, such that $\operatorname{dom}\left(H_{\eta}\right)=X_{\eta}$ and $f(\eta)<H_{\eta}\left(x_{\eta}^{*}\right)$ for all large $\eta<\lambda$.

(2) Suppose that in $V[E][G], f \in \prod_{\xi<\lambda} f_{\nu}^{\xi}\left(\tau_{\xi}\right)^{+}$. Then there is a sequence of functions $\left\langle H_{\eta} \mid \eta<\lambda\right\rangle$ in $V[E]$, such that $\operatorname{dom}\left(H_{\eta}\right)=X_{\eta}$ and $f(\eta)<H_{\eta}\left(x_{\eta}^{*}\right)$ for all large $\eta<\lambda$.

Proof of Proposition 8. The proof is an adaptation of the arguments in [1] and [6], so we only outline the main points. For more details, see sections 4 and 5 of $[6]$. Define in $V[E][G],\left\langle g_{\beta} \mid \beta<\mu\right\rangle$ in $\prod_{\xi<\lambda} f_{\kappa_{\xi}^{+}}^{\xi}\left(\tau_{\xi}\right)$ by $g_{\gamma}(\xi)=$ $f_{G_{\gamma}(\xi)}^{\xi}\left(\tau_{\xi}\right)$. By Proposition 9, it follows that the functions are increasing in the eventual domination order. Also, by the bounding lemma (1), we get that the sequence is cofinal. So, $\left\langle g_{\beta} \mid \beta<\mu\right\rangle$ is a scale.

Using the fact that all of the measures are $\tau$-complete and Remark 6 , we get that if a point of cofinality $\tau$ is bad in $V[E]$ for $\left\langle G_{\beta} \mid \beta<\mu\right\rangle$, then it is bad in $V[E][G]$ for $\left\langle g_{\beta} \mid \beta<\mu\right\rangle$ (and still has cofinality $\tau$ in $V[E][G]$ ). Since $\mathbb{P}$ has the $\mu$-chain condition, and so preserves stationary sets, it follows that $\left\langle g_{\beta} \mid \beta<\mu\right\rangle$ is a bad scale.

Next we describe the very good scale. In $V[E][G]$, define $\left\langle h_{\gamma} \mid \gamma<\mu\right\rangle$ in $\prod_{\xi<\lambda} f_{\nu}^{\xi}\left(\tau_{\xi}\right)^{+}$, by $h_{\gamma}(\xi)=f_{\gamma}^{\xi}\left(\tau_{\xi}\right)$. By Proposition 9 , the bounding lemma $(2)$, and the completeness of the measures, we get that this is a very good scale. 


\section{THE TREE PROPERTY}

It remains to show that the tree property holds. Recall that we forced over $V$ with $A=A d d\left(\kappa, \nu^{++}\right)$to get $V[E]$. Let $\dot{T}$ in $V[E]$ be a $\mathbb{P}$ - name for a $\nu^{+}$tree with levels of size at most $\kappa$, such that this is forced by the empty condition. Furthermore we may assume that the empty condition forces that the elements of the $\alpha$-th level of $\dot{T}$ are elements of $\{\alpha\} \times \kappa$ for $\alpha<\nu^{+}$. We will show that $T=\dot{T}_{G}$ has a cofinal branch in $V[E][G]$. The proof is motivated by Neeman [5].

Lemma 11. There is $\vec{\eta} \in \lambda^{<\omega}$ and an unbounded $I \subset \nu^{+}$(in $V[E]$ ), such that for all $\alpha<\beta$ in $I$, there are $\xi, \delta<\kappa$ and a condition $q=\langle g, H\rangle$ with $\operatorname{dom}(g)=\vec{\eta}$, such that $q \Vdash\langle\alpha, \xi\rangle<_{\dot{T}}\langle\beta, \delta\rangle$.

Proof. Let $j: V[E] \rightarrow M$ be $\nu^{+}$supercompact embedding with critical point $\kappa$. Let $G^{*}$ be $j(\mathbb{P})$ - generic over $M$, such that the generic sequence determined by $G^{*}$ is above $\nu^{+}$. In particular, if $\left\langle x_{\xi}^{*} \mid \xi<\lambda\right\rangle$ is the generic sequence, where each $x_{\xi}^{*} \in \mathcal{P}_{j(\kappa)}\left(j\left(\kappa_{\xi}\right)\right)$, and $\tau_{\xi}^{*}=j(\kappa) \cap x_{\xi}^{*}$ for each $\xi, G^{*}$ is chosen to be such that $\nu^{+}<\tau_{0}^{*}$. Set $T^{*}=j(\dot{T})_{G^{*}}$.

Let $\gamma$ be such that $\sup j^{\prime \prime} \nu^{+}<\gamma<j\left(\nu^{+}\right)$. Such a $\gamma$ exists since $M$ is closed under $\nu^{+}$-sequences. Working in $M\left[G^{*}\right]$, fix a node $u \in T^{*}$ of level $\gamma$. Then for all $\alpha<\nu^{+}$let $\xi_{\alpha}<j(\kappa)$ be such that $\left\langle j(\alpha), \xi_{\alpha}\right\rangle<_{T^{*}} u$, and let $p_{\alpha} \in G^{*}$ be such that $p_{\alpha} \Vdash\left\langle j(\alpha), \xi_{\alpha}\right\rangle<_{j(\dot{T})} \dot{u}$.

Since the generic sequence determined by $G^{*}$ is above $\nu^{+}$, we have that $\nu^{+}$is preserved in $M\left[G^{*}\right]$ and remains regular. So, there is an unbounded $I^{*} \subset \nu^{+}$in $M\left[G^{*}\right]$ and a fixed $\vec{\eta} \in \lambda^{<\omega}$, such that for all $\alpha \in I^{*}, p_{\alpha}=$ $\left\langle g_{\alpha}, H_{\alpha}\right\rangle$ where the domain of $g_{\alpha}$ is $\vec{\eta}$. Let $b$ be a stem with this domain such that there is a condition in $G^{*}$ with stem $b$.

Define $I=\left\{\alpha<\nu^{+} \mid \exists p \in j(\mathbb{P}) \operatorname{stem}(p)=b\right.$ and $\exists \xi<j(\kappa) p \Vdash$ $\left.\langle\alpha, \xi\rangle<_{j(\dot{T})} \dot{u}\right\}$. Then $I \in V[E]$ and $I^{*} \subset I$, so $I$ is unbounded. Any two conditions with the same stem are compatible, so by elementarity of $j$ and since $j(\dot{T})$ is forced to be a tree, we have that $I$ is as desired.

Lemma 12. There is, in $V[E]$, an unbounded set $J \subset \nu^{+}$, a pair $\langle\bar{g}, \bar{H}\rangle$ and a sequence of nodes $\left\langle u_{\alpha} \mid \alpha \in J\right\rangle$, such that, setting $\operatorname{dom}(\bar{g})=\vec{\eta}$ and $\eta_{0}=\max (\vec{\eta})$, we have that $\bar{H}$ has domain $\eta_{0} \backslash \vec{\eta}$ and for all $\alpha<\beta$ in $J$ there is a condition $p$ such that:

- $\operatorname{stem}(p)=\bar{g}, p\left\lceil\left(\eta_{0}+1\right)=\langle\bar{g}, \bar{H}\rangle\right.$,

- $p \Vdash u_{\alpha}<_{\dot{T}} u_{\beta}$.

Proof. Fix $\vec{\eta}$ and $I$ as in the conclusion of the last lemma, and let $\eta_{0}=$ $\max (\vec{\eta})$. Let $\bar{j}: V \rightarrow N$ be a $\nu^{+}$- supercompact embedding with critical point $\kappa_{\eta_{0}+1}$. Using standard arguments, extend $\bar{j}$ to $j: V[E] \rightarrow N\left[E^{*}\right]$ where $j \in V[E][F]$, for $F-A d d\left(\kappa_{0}, j\left(\nu^{++}\right)\right)$- generic over $V[E]$.

Let $\gamma \in j(I)$ be such that $\sup \left(j^{\prime \prime} \nu^{+}\right)<\gamma<j\left(\nu^{+}\right)$. By elementarity for all $\alpha \in I$ we can fix $\xi_{\alpha}, \delta_{\alpha}<\kappa_{0}$ and $p_{\alpha}=\left\langle g_{\alpha}, H_{\alpha}\right\rangle \in j(\mathbb{P})$ with domain of $g_{\alpha}$ equal to $\vec{\eta}$, such that $p_{\alpha} \Vdash\left\langle j(\alpha), \xi_{\alpha}\right\rangle<_{j(\dot{T})}\left\langle\gamma, \delta_{\alpha}\right\rangle$. I is cofinal in $\nu^{+}$ 
and the number of possibilities for the part of the conditions below $\eta_{0}+1$ is less than $\kappa_{\eta_{0}+1}$. It follows that there is a cofinal $J \subset I$ in $V[E][F]$, fixed $\xi, \delta<\kappa_{0}$, and a fixed $\langle\bar{g}, \bar{H}\rangle$ such that for all $\alpha \in J, \delta_{\alpha}=\delta, \xi_{\alpha}=\xi$, and $p_{\alpha} \uparrow\left(\eta_{0}+1\right)=\langle\bar{g}, \bar{H}\rangle$. Then for all $\alpha, \beta \in J$ with $\alpha<\beta$, there is a condition $p \in j(\mathbb{P})$ with stem $\bar{g}$ and $p \nmid\left(\eta_{0}+1\right)=\langle\bar{g}, \bar{H}\rangle$, such that $p \Vdash\langle j(\alpha), \xi\rangle<_{j(\dot{T})}\langle j(\beta), \xi\rangle$. Since $j(\langle\bar{g}, \bar{H}\rangle)=\langle\bar{g}, \bar{H}\rangle$, by elementarity, there is a condition $p \in \mathbb{P}$ such that $p\left\lceil\left(\eta_{0}+1\right)=\langle\bar{g}, \bar{H}\rangle\right.$ and $p \Vdash\langle\alpha, \xi\rangle<_{\dot{T}}\langle\beta, \xi\rangle$.

In $V[E]$ let $a=\left\{\langle g, H, \alpha, \beta, \xi\rangle \mid(\exists p \in \mathbb{P})\left(\operatorname{stem}(p)=g, p\left\lceil\left(\eta_{0}+1\right)=\right.\right.\right.$ $\left.\left.\langle g, H\rangle, p \Vdash\langle\alpha, \xi\rangle<_{\dot{T}}\langle\beta, \xi\rangle\right)\right\}$. Note that $a$ can be coded by a subset of $\nu^{+}$. So far we have shown that $V[E][F] \mid=(\exists g, H, J, \xi) \phi\left(a, g, H, J, \xi, \nu^{+}\right)$, where $\phi\left(a, g, H, J, \xi, \nu^{+}\right)=$" $J$ is an unbounded subset of $\nu^{+}$and for all $\alpha, \beta \in J$ with $\alpha<\beta,\langle g, H, \alpha, \beta, \xi\rangle \in a$ ".

Claim 13. $V[E] \models(\exists g, H, J, \xi) \phi\left(a, g, H, J, \xi, \nu^{+}\right)$

Proof. See Neeman [5]. The proof uses that $a$ can be coded by a subset of $\nu^{+}$and the absoluteness of $\phi$.

Fix $\langle\bar{g}, \bar{H}\rangle, J$, and $u_{\alpha}=\langle\alpha, \xi\rangle$ for $\alpha \in J$ as in the conclusion of the above lemma. For a stem $g$ and a formula $\phi$, we say that $g \Vdash^{*} \phi$ iff there is a condition $p$ with stem $g$ such that $p \Vdash \phi$. By the Prikry property it follows that for all $g$ and $\phi$, either $g \Vdash^{*} \phi$ or $g \Vdash^{*} \neg \phi$.

The following proposition is due to Neeman [5].

Proposition 14. Suppose that $M$ is a model of $Z F C, S$ is a tree of height $\theta$ in $M$, and $\mathbb{B} \in M$ is a poset such that $\mathbb{B} \times \mathbb{B}$ has the $\operatorname{cf}(\theta)$-chain condition and a power $\mathbb{B}^{|S|^{+}}$does not collapse $|S|^{+}$. Then $\mathbb{B}$ does not add a branch through $S$.

Lemma 15. Suppose that $g$ is a stem, $L \subset \nu^{+}$is unbounded, and for all $\alpha<\beta$ with $\alpha, \beta \in L, g \mathbb{\Vdash}^{*} u_{\alpha}<_{\dot{T}} u_{\beta}$. Let $\eta>\max (\operatorname{dom}(g))$.

Then there are $\rho<\nu^{+}$and sets $\left\langle A_{\alpha}: \alpha \in L \backslash \rho\right\rangle$ such that:

(1) each $A_{\alpha} \in U_{\eta}$,

(2) for all $\alpha<\beta$ in $L \backslash \rho$, for all $x \in A_{\alpha} \cap A_{\beta}$,

$$
g \frown\langle\eta, x\rangle \Vdash^{*} u_{\alpha}<_{\dot{T}} u_{\beta} .
$$

Proof. The proof follows closely the argument given in [5].

Let $\bar{j}: V \rightarrow N$ be a $\nu^{+}$- supercompact embedding with critical point $\kappa_{\eta+1}$. As in the previous lemma, extend $\bar{j}$ to $j: V[E] \rightarrow N\left[E^{*}\right]$ where $j \in V[E][F]$, for $F-A d d\left(\kappa, j\left(\nu^{++}\right)\right)$- generic over $V[E]$. Let $\gamma \in j(L)$ be such that $j^{\text {" }} \nu^{+}<\gamma<j\left(\nu^{+}\right)$. We write $u_{\gamma}$ for the $\gamma^{\text {th }}$ member of the sequence $j\left(\left\langle u_{\alpha}: \alpha \in L\right\rangle\right)$. Note that $j(g)=g$. Then working in $V[E][F]$, by elementarity we can find conditions $\left\langle r_{\alpha}: \alpha \in L\right\rangle$, such that each $r_{\alpha} \in j(\mathbb{P})$, the stem of each $r_{\alpha}$ is $g$, and

$$
r_{\alpha} \Vdash_{j(P)} j\left(u_{\alpha}\right)<_{j(\dot{T})} u_{\gamma} .
$$


For each $\alpha \in L$, denote $r_{\alpha}=\left\langle g, H_{\alpha}\right\rangle$. Define $\alpha \mapsto A_{\alpha}^{*}$ (in $V[E][F]$ ) by $A_{\alpha}^{*}=H_{\alpha}(\eta) \in j\left(U_{\eta}\right)$. Note that although they have the same domain, $U_{\eta} \neq j\left(U_{\eta}\right)$. Actually, $\mathcal{P}\left(\mathcal{P}_{\kappa}\left(\kappa_{\eta}\right)\right)^{V[E]} \neq \mathcal{P}\left(\mathcal{P}_{\kappa}\left(\kappa_{\eta}\right)\right)^{V[E][F]}$, as the cardinality of the latter is $j\left(\nu^{++}\right)$.

Equation 1 implies that for all $x \in A_{\alpha}^{*}$ we have that over $N\left[E^{*}\right]$.

$$
g \frown\langle\eta, x\rangle \Vdash_{j(\mathbb{P})}^{*} j\left(u_{\alpha}\right)<_{j(\dot{T})} u_{\gamma}
$$

For $x \in \mathcal{P}_{\kappa}\left(\kappa_{\eta}\right)$, let $L_{x}=\left\{\alpha \in L \mid g \frown\langle\eta, x\rangle \Vdash_{j(\mathbb{P})}^{*} j\left(u_{\alpha}\right)<_{j(\dot{T})} u_{\gamma}\right\}$. Note that for all $\alpha \in L$, if $x \in A_{\alpha}^{*}$, then $\alpha \in L_{x}$.

Claim 16. If $L_{x}$ is unbounded in $\nu^{+}$, then $L_{x} \in V[E]$.

Proof. Suppose that $L_{x}$ is unbounded in $\nu^{+}$.

Subclaim 17. For all $\alpha, \beta$ in $L$ with $\alpha<\beta$ and $\beta \in L_{x}$, we have that $\alpha \in L_{x}$ iff $g \frown\langle\eta, x\rangle \Vdash^{*} u_{\alpha}<_{\dot{T}} u_{\beta}$.

Proof. Let $\alpha, \beta$ be as above. So, $g \frown\langle\eta, x\rangle \Vdash_{j(\mathbb{P})}^{*} j\left(u_{\beta}\right)<_{j(\dot{T})} u_{\gamma}$. Then $\alpha \in L_{x}$ iff $g \frown\langle\eta, x\rangle \Vdash_{j(\mathbb{P})}^{*} j\left(u_{\alpha}\right)<_{j(\dot{T})} u_{\gamma}$ iff $g \frown\langle\eta, x\rangle \Vdash_{j(\mathbb{P})}^{*} j\left(u_{\alpha}\right)<_{j(\dot{T})} j\left(u_{\beta}\right)$ iff $g \frown\langle\eta, x\rangle \Vdash_{\mathbb{P}}^{*} u_{\alpha}<_{\dot{T}} u_{\beta}$.

Let $S$ be the tree of attempts to construct $L_{x}$. I.e. $S$ is the set of all bounded $v: \nu^{+} \rightarrow L$ such that:

(1) $v$ is increasing,

(2) for all $\alpha, \beta$ in $L$ with $\alpha<\beta$ and $\beta \in \operatorname{ran}(v)$, we have that $\alpha \in \operatorname{ran}(b)$ iff $g \frown\langle\eta, x\rangle \Vdash^{*} u_{\alpha}<_{\dot{T}} u_{\beta}$.

Then if we let $v^{*}: \nu^{+} \rightarrow L$ enumerate $L_{x}$, we have an unbounded branch of $S$. Here we use that all initial segments of $L_{x}$ are in $V[E]$. Applying Proposition 14 for $M=V[E], \theta=\nu^{+}, \mathbb{B}=\operatorname{Add}\left(\kappa, j\left(\nu^{++}\right)\right)$, and $S$, we get that $L_{x} \in V[E]$.

For $x \in \mathcal{P}_{\kappa}\left(\kappa_{\eta}\right)$, let $K_{x}=\left\{C \in V[E] \mid C\right.$ is cofinal in $\nu^{+}$and there is a $b \in \operatorname{Add}\left(\kappa, j\left(\nu^{++}\right)\right)$with $\left.b \Vdash \dot{L_{x}}=C\right\}$ if $g \frown\langle\eta, x\rangle$ is a stem, and $K_{x}=\emptyset$ otherwise. Then each $K_{x} \in V[E]$ and since $A d d\left(\kappa, j\left(\nu^{++}\right)\right)$has the $\kappa^{+}$chain condition, we have that card $\left(K_{x}\right) \leq \kappa$.

Claim 18. For $C \in K_{x}$ and $\alpha<\beta<\nu^{+}$such that both $\alpha$ and $\beta$ are in $L$ and $\beta \in C$, we have that $\alpha \in C$ iff $g^{\frown}\langle\eta, x\rangle \Vdash^{*} u_{\alpha}<_{\dot{T}} u_{\beta}$.

Proof. similar as in the subclaim above.

From the above claim it follows that any distinct $C_{1}, C_{2}$ in $K_{x}$ are disjoint on a tail. For every $x$, and $C_{1}, C_{2}$ in $K_{x}$ fix $\rho_{x, C_{1}, C_{2}}$ to be such that above it, $C_{1}$ and $C_{2}$ are disjoint. Let $\rho=\sup \left\{\rho_{x, C_{1}, C_{2}} \mid x \in \mathcal{P}_{\kappa}\left(\kappa_{\eta}\right), C_{1}, C_{2} \in K_{x}\right\}$. Then $\rho<\nu^{+}$, and for all $x$ and $\alpha \in L \backslash \rho$, there is at most one $C \in K_{x}$ with $\alpha \in C$. Define $f(x, \alpha)$ to be this unique $C \in K_{x}$ if it exists and undefined 
otherwise. For $\alpha \in L \backslash \rho$ let $G_{\alpha}=\left\{x \in \mathcal{P}_{\kappa}\left(\kappa_{\eta}\right) \mid f(x, \alpha)\right.$ is defined $\}$. Also for $\alpha, \alpha^{\prime} \in L \backslash \rho$, define $G_{\alpha, \alpha^{\prime}}=\left\{x \in G_{\alpha} \cap G_{\alpha^{\prime}} \mid f(x, \alpha)=f\left(x, \alpha^{\prime}\right)\right\}$.

Claim 19. (1) For each $\alpha, G_{\alpha} \in U_{\eta}$

(2) For each $\alpha, \alpha^{\prime}, G_{\alpha, \alpha^{\prime}} \in U_{\eta}$

Proof. Otherwise, for some $\alpha \in L \backslash \rho, Y=\left\{x \in \mathcal{P}_{\kappa}\left(\kappa_{\eta}\right) \mid f(x, \alpha)\right.$ is not defined $\} \in U_{\eta}$. Since $j(Y)=Y \in j\left(U_{\eta}\right)$, we have that for all $\beta \in L$, we can fix an element $x_{\beta} \in A_{\alpha}^{*} \cap A_{\beta}^{*} \cap Y$. L is unbounded in $\nu^{+}$, so there is some unbounded $U \subset L$ and $x \in \mathcal{P}_{\kappa}\left(\kappa_{\eta}\right)$, such that for all $\beta \in U, x_{\beta}=x$.

Now, for all $\beta \in U$, since $x \in A_{\beta}^{*}$, we have that $g \frown\langle\eta, x\rangle \Vdash^{*} j\left(u_{\beta}\right)<_{j(\dot{T})}$ $u_{\gamma}$, so $\beta \in L_{x}$. I.e $U \subset L_{x}$, and so $L_{x}$ is unbounded and thus it is in $V[E]$. Also, since $x \in A_{\alpha}^{*}$, it follows that $g^{\frown}\langle\eta, x\rangle \Vdash^{*} j\left(u_{\alpha}\right)<_{j(\dot{T})} u_{\gamma}$, and so $\alpha \in L_{x}$. In particular, $\alpha \in L_{x} \in K_{x}$, therefore $f(x, \alpha)$ is defined. But $x \in Y$, contradiction.

The proof of (2) is similar.

Let $\alpha_{0}$ be the least element in $L \backslash \rho$. Define $A_{\alpha}=G_{\alpha_{0}, \alpha}$. The sets $\left\langle A_{\alpha}: \alpha \in L \backslash \rho\right\rangle$ are as desired.

Lemma 20. There are $\rho<\nu^{+}$and conditions $\left\langle p_{\alpha}: \alpha \in J \backslash \rho\right\rangle$ such that:

(1) each $p_{\alpha}$ has stem $\bar{g}$ and $p_{\alpha} \uparrow\left(\eta_{0}+1\right)=\langle\bar{g}, \bar{H}\rangle$,

(2) for all $\alpha<\beta$ in $J \backslash \rho, p_{\alpha} \wedge p_{\beta} \Vdash u_{\alpha}<_{\dot{T}} u_{\beta}$.

Here $p \wedge q$ denotes the weakest extension of $p$ and $q$.

Proof. Recall that $\langle\bar{g}, \bar{H}\rangle, \eta_{0}=\max (\operatorname{dom}(g)), J$, and $u_{\alpha}=\langle\alpha, \xi\rangle$ are given by Lemma 12. I.e. $\bar{H}$ has domain $\eta_{0} \backslash \vec{\eta}$ and for all $\alpha<\beta$ in $J$ there is a condition $p$ such that the stem of $p$ is $\bar{g}, p\left\lceil\left(\eta_{0}+1\right)=\langle\bar{g}, \bar{H}\rangle\right.$, and $p \Vdash u_{\alpha}<_{\dot{T}} u_{\beta}$.

First we make some remarks on taking diagonal intersections. Let $\eta<$ $\lambda$, let $S$ be a set of stems whose domain has maximum below $\eta$, and let $\left\langle A^{g} \mid g \in S\right\rangle$ be a sequence of $U_{\eta^{-}}$measure one sets. For a stem $g$ in $S$ and $z \in \mathcal{P}_{\kappa}\left(\kappa_{\eta}\right)$, we write $g \prec z$ to denote that $g(\max \operatorname{dom}(g)) \prec z$, i.e. that $|g(\max \operatorname{dom}(g))|<\kappa_{z}$ and $g(\max \operatorname{dom}(g)) \subset z$. Note that $g \prec z$ iff $g \frown\langle\eta, z\rangle$ is a stem. Then $A=\triangle_{g \in S} A^{g}=\left\{z \in \mathcal{P}_{\kappa}\left(\kappa_{\eta}\right) \mid z \in \bigcap_{g \prec z} A^{g}\right\}$ is the diagonal intersection of $\left\langle A^{g} \mid g \in S\right\rangle$ and $A \in U_{\eta}$.

Let $g \supset \bar{g}$ be a stem with $\max (\operatorname{dom}(g))=\eta$. We say that $g$ is compatible with $\bar{H}$ if for all $\xi \in \operatorname{dom}(g) \cap \eta_{0}$ with $\xi \notin \operatorname{dom}(\bar{g}), g(\xi) \in \bar{H}(\xi)$. Also for $\eta^{\prime} \geq \eta$, we say that $g$ is compatible with $\left\langle B_{\xi} \mid \eta_{0}<\xi \leq \eta^{\prime}\right\rangle$, where each $B_{\xi} \in$ $U_{\xi}$, if for all $\eta_{0}<\xi \in \operatorname{dom}(g), g(\xi) \in B_{\xi}$. Note that if $\max (\operatorname{dom}(g))=\eta_{0}$, then $g$ is vacuously compatible with any sequence $\left\langle B_{\xi} \mid \eta_{0}<\xi \leq \eta^{\prime}\right\rangle$. We will define sequences $\left\langle\rho_{\eta} \mid \eta_{0}<\eta<\lambda\right\rangle$, and $\left\langle A_{\alpha}(\eta) \mid \alpha \in J \backslash \rho_{\eta}, \eta_{0}<\eta<\lambda\right\rangle$ by induction on $\eta$, such that each $A_{\alpha}(\eta) \in U_{\eta}$ and for all $\eta_{0} \leq \eta<\lambda$ we have: 
$(\dagger)_{\eta}$ For all stems $g \supset \bar{g}$ with $\max (\operatorname{dom}(g))=\eta$, and for all $\alpha<\beta$ in $J \backslash \rho_{\eta}$, if $g$ is compatible with $\bar{H},\left\langle A_{\alpha}(\xi) \mid \eta_{0}<\xi \leq \eta\right\rangle$, and $\left\langle A_{\beta}(\xi) \mid \eta_{0}<\xi \leq \eta\right\rangle$, then $g \Vdash^{*} u_{\alpha}<_{\dot{T}} u_{\beta}$.

Note that $(\dagger)_{\eta_{0}}$ holds by Lemma 12.

Suppose $\eta_{0}<\eta<\lambda$ and suppose that we have defined $\rho_{\xi}$ and $A_{\alpha}(\xi)$ for all $\xi<\eta, \alpha \in J \backslash \rho_{\xi}$ such that $(\dagger)_{\xi}$ holds for all $\eta_{0} \leq \xi<\eta$.

For a stem $g \supset \bar{g}$ with $\max (\operatorname{dom}(g))<\eta$ set $J^{g}=\left\{\alpha \in J \backslash \sup _{\eta_{0}<\xi<\eta} \rho_{\xi} \mid g\right.$ is compatible with $\left.\left\langle A_{\alpha}(\xi) \mid \eta_{0}<\xi \leq \max (\operatorname{dom}(g))\right\rangle\right\}$ if $\eta>\eta_{0}+1$, and if $\eta=\eta_{0}+1$, we set $J^{g}=J$. Define a function $g \mapsto \rho^{g}$ on stems extending $\bar{g}$ and compatible with $\bar{H}$ whose domain has maximum below $\eta$ as follows:

- if $J^{g}$ is bounded in $\nu^{+}$, let $\rho^{g}<\nu^{+}$be a bound,

- otherwise, let $\rho^{g}$ and $\left\langle A_{\alpha}^{g} \mid \alpha \in J^{g} \backslash \rho^{g}\right\rangle$, be given by the previous lemma applied to $g$ and $J^{g}$ (here we use $(\dagger)_{\max (\operatorname{dom}(g) \text { ) }}$ to get the assumptions of the lemma). Then, we have that:

- each $A_{\alpha}^{g} \in U_{\eta}$,

- for all $\alpha<\beta$ in $J \backslash \rho^{g}$, for all $y \in A_{\alpha}^{g} \cap A_{\beta}^{g}$,

$$
g \frown\langle\eta, y\rangle \Vdash^{*} u_{\alpha}<_{\dot{T}} u_{\beta} .
$$

Let $\rho_{\eta}=\sup _{g} \rho^{g}$. Note that the number of all possible such stems is less that $\nu^{+}$, so $\rho_{\eta}<\nu^{+}$. For each $\alpha \in J \backslash \rho_{\eta}$, define $H_{\alpha}=\{g \mid g$ is a stem, $g \supset \bar{g}, \max (\operatorname{dom}(g))<\eta, g$ is compatible with $\bar{H},\left\langle A_{\alpha}(\xi)\right| \eta_{0}<\xi \leq$ $\max (\operatorname{dom}(g))\rangle\}$. Then since $\alpha>\rho_{\eta}$, for each $g \in H_{\alpha}, A_{\alpha}^{g}$ is defined. For $\alpha \in J \backslash \rho_{\eta}$ define

$$
A_{\alpha}(\eta)=\triangle_{g \in H_{\alpha}} A_{\alpha}^{g}
$$

Now we have to verify that $(\dagger)_{\eta}$ holds: suppose that $\alpha<\beta$ are in $J \backslash \rho_{\eta}$ and $g^{\prime} \supset \bar{g}$ is compatible with $\left\langle A_{\alpha}(\xi) \mid \eta_{0}<\xi \leq \eta\right\rangle,\left\langle A_{\beta}(\xi) \mid \eta_{0}<\xi \leq \eta\right\rangle$, $\bar{H}$, and $\max \left(\operatorname{dom}\left(g^{\prime}\right)\right)=\eta$. Then for some $y, g^{\prime}=g \cup\langle\eta, y\rangle$. Since $g^{\prime}$ is compatible with $\left\langle A_{\alpha}(\xi) \mid \eta_{0}<\xi \leq \eta\right\rangle$ and $\left\langle A_{\beta}(\xi) \mid \eta_{0}<\xi \leq \eta\right\rangle$, we have that $y \in A_{\alpha}(\eta) \cap A_{\beta}(\eta)$. Therefore since $g \prec y$, we have that $y \in A_{\alpha}^{g} \cap A_{\beta}^{g}$. Since $\alpha, \beta \in J^{g}$ and $\alpha, \beta \geq \rho_{\eta} \geq \rho^{g}$, it follows that $\rho^{g}, A_{\alpha}^{g}$, and $A_{\beta}^{g}$ were given by Lemma 15, so $g \frown\langle\eta, y\rangle \Vdash^{*} u_{\alpha}<_{\dot{T}} u_{\beta}$ as desired.

Let $\rho=\sup \left\{\rho_{\eta} \mid \eta_{0}<\eta<\lambda\right\}$. Define $p_{\alpha}$ for $\alpha<J \backslash \rho$ by:

- $p_{\alpha} \uparrow\left(\eta_{0}+1\right)=\langle\bar{g}, \bar{H}\rangle$

- $p_{\alpha}(\eta)=A_{\alpha}(\eta)$, for $\eta_{0}<\eta<\lambda$

Now suppose that $q \leq p_{\alpha} \wedge p_{\beta}$. Let $g$ be the stem of $q$ and let $\eta=$ $\max (\operatorname{dom}(g))$. Then $g$ is compatible with $\bar{H},\left\langle A_{\alpha}(\xi) \mid \eta_{0}<\xi \leq \eta\right\rangle$, and $\left\langle A_{\beta}(\xi) \mid \eta_{0}<\xi \leq \eta\right\rangle$, so by $(\dagger)_{\eta}$ we have that $g \Vdash^{*} u_{\alpha}<_{\dot{T}} u_{\beta}$. Since any two conditions with the same stem are compatible, it follows that $q \forall u_{\alpha} \nless_{\dot{T}} u_{\beta}$. So, $p_{\alpha} \wedge p_{\beta} \Vdash u_{\alpha}<_{\dot{T}} u_{\beta}$ as desired.

Lastly, we show that $\left\{u_{\alpha} \mid p_{\alpha} \in G\right\}$ is an unbounded branch of $T$. It suffices to prove the following:

Proposition 21. $B=\left\{\alpha<\nu^{+} \mid p_{\alpha} \in G\right\}$ is unbounded. 
Proof. Otherwise, let $q \in G$ be such that $q \Vdash \dot{B}$ is bounded. Since both Lemma 11 and Lemma 12 can be done below any condition, we may assume that (by strengthening $q$ if necessary) $\operatorname{stem}(q)=\bar{g}$. $\mathbb{P}$ has the $\nu^{+}$chain condition, so for some $\alpha<\nu^{+}, q \Vdash \dot{B} \subset \alpha$. Let $\beta \in J \backslash \alpha$, and let $r$ be a common extension of $q$ and $p_{\beta}$. Then on one hand we have that $r \Vdash p_{\beta} \in \dot{G}$, but also $r \Vdash u_{\beta} \notin \dot{B}$. Contradiction.

Then $\left\{u_{\alpha} \mid \alpha \in B\right\}$ is an unbounded branch of $T$. This completes the proof of the tree property.

\section{REFERENCES}

[1] James Cummings and Matthew Foreman, Diagonal Prikry extensions, J. of Symbolic Logic, vol. 75(2010), no. 4, pp. 1383-1402.

[2] Moti Gitik and Assaf Sharon, On SCH and the approachability property, Proc. of the $\boldsymbol{A M S}$, vol. 136 (2008), pp. 311-320

[3] Richard LaVer, Making the supercompactness of $\kappa$ indestructible under $\kappa$-directed closed forcing, Israel J. of Math., vol. 29(1978), no. 4, pp. 385-388.

[4] Menachem Magidor and Saharon Shelah, The tree property at successors of singular cardinals., Arch. Math. Logic, vol. 35 (1996), no. 5-6, pp. 385-404

[5] Itay Neeman, Aronszajn trees and the failure of the singular cardinal hypothesis, $\boldsymbol{J}$. of Mathematical Logic vol. 9 (2009, published 2010) pp. 139-157.

[6] Dima Sinapova, A model for a very good scale and a bad scale., J. Symbolic Logic, 73(4):1361-1372, 2008

[7] Robert M. Solovay, William N. Reinhardt and Akihiro Kanamori, Strong axioms of infinity and elementary embeddings., Ann. Math. Logic, vol. 13(1978), no. 1 , pp. $73-116$.

Department of Mathematics, University of California Irvine, Irvine, CA 92697-3875, U.S.A

E-mail address: dsinapov@math.uci.edu 